\title{
Síndrome de Loeys-Dietz, una mutación en el gen TGFBR2, primer reporte en el suroccidente colombiano
}

\author{
Loeys Dietz syndrome, a mutation in TGFBR2 gene,
}

first report in the colombian suroccident

María A. Acosta-Aragón $P h D^{1}$,
Marco F. Sierra-Zúñiga $M D^{2}$

Resumen: el síndrome de Loeys-Dietz es una rara enfermedad genética, autosómica dominante, con hábito marfanoide, que pertenece a un subconjunto de enfermedades del tejido conectivo con afectación esquelética, ocular y cardiovascular, principalmente. El desarrollo de aneurismas es característico en esta patología. El síndrome de Loeys-Dietz es causado por mutaciones en los genes TGFBR1, TGF$B R 2, T G F B 2, T G F B 3$ Y SMAD3. En este manuscrito se describe el caso clínico de un paciente masculino, de 22 meses de vida, con una dilatación importante de la raíz aórtica y arco aórtico elongado cuya prueba molecular confirma el diagnóstico de síndrome de Loeys-Dietz, asociado a una mutación en el gen TGFBR2. Este corresponde al primer caso reportado en el suroccidente colombiano.

Palabras clave: síndrome de Loeys-Dietz, receptor del factor de crecimiento transformador beta tipo 2, mutación, manejo de caso.

Acosta-Aragón MA, Sierra-Zúñiga MF. Síndrome de Loeys-Dietz, una mutación en el gen TGFBR2, primer reporte en el suroccidente colombiano. Medicina \& Laboratorio 2017; 23: 187-194.

E I síndrome de Loeys-Dietz fue conocido como el síndrome de Furlong, desde 1987 [1] hasta 2005, momento en el que se establecen claramente sus características clínicas y moleculares por dos genetistas pediatras, Bart Loeys y Harry Dietz, de la universidad John Hopkins de Estados Unidos [2]. El síndrome de Loeys-Dietz es una rara enfermedad genética, autosómica dominante [3], pero que se puede encontrar como una mutación de novo hasta en el $75 \%$ de los casos [4]. Esta es una patología con hábito marfanoide, la cual pertenece a un subconjunto de enfermedades del tejido conectivo, con afectación esquelética, ocular y cardiovascular, principalmente $[3,5]$.

\footnotetext{
${ }^{1}$ Médica y Cirujana, MSc en Biología, área Genética Clínica, PhD en Genética de Poblaciones Humanas y Genética Forense. Profesora titular, Departamento de Pediatría, Facultad de Ciencias de la Salud, Universidad del Cauca. Popayán, Colombia. Correo electrónico: morin1924@gmail.com ${ }^{2}$ Médico y cirujano, residente en Pediatría, Departamento de Pediatría, Facultad de Ciencias de la

Salud, Universidad del Cauca. Popayán, Colombia.

Conflicto de intereses: Ios autores declaran que no tienen conflicto de intereses Medicina \& Laboratorio 2017; 23: 187-194

Módulo 21 (Casos clínicos), número 19. Editora Médica Colombiana S.A. 2017 Recibido el 02 de abril de 2017; aceptado el 29 de abril de 2017
} 
Esta enfermedad se puede manifestar en cualquier momento de la vida y su gravedad es variable [4]; se caracteriza por el desarrollo de aneurismas o disecciones arteriales a nivel cerebral, torácico o abdominal en edades tempranas; además de diversas manifestaciones esqueléticas como aranodactilia, hiperlaxitud articular, contracturas congénitas de los dedos y pie equinovaro $[3,5]$. Otras manifestaciones son la presencia de hipertelorismo, paladar hendido y úvula bífida [6]. Por lo general, el coeficiente intelectual no se ve comprometido $[3,7]$.

El cuadro clínico del síndrome de Loeys-Dietz guarda muchas similitudes con el presentado en el síndrome de Marfan y el síndrome de Ehlers-Danlos [5,7]. Entre los diagnósticos diferenciales se incluyen, además, la aracnodactilia contractural congénita y el síndrome de Shprintzen-Goldberg $[7,8]$. La presencia de úvula bífida permite el diagnóstico diferencial con el síndrome de Marfan. Cabe anotar que la severidad de los trastornos cardiovasculares es mayor en el síndrome de Loeys-Dietz que en el síndrome de Marfan [7]. El diagnóstico se realiza mediante los hallazgos característicos al examen físico y estudios genéticos moleculares [9].

El síndrome de Loeys-Dietz es causado por mutaciones en los genes TGFBR1, TGFBR2, TGFB2, TGFB3 y SMAD3 [4,9-12] y se clasifica en cinco tipos. El tipo 1 presenta manifestaciones craneofaciales (75\%) y una tríada característica comprendida por tortuosidad arterial, hipertelorismo ocular y úvula bífida o paladar hendido. El tipo 2 presenta manifestaciones cutáneas (25\%), sin craneosinostosis ni paladar hendido, pero sí úvula bífida [7]. Su origen se debe a una mutación en los genes TGFBR1 (9q22.33) y TGFBR2 (3p24.1), respectivamente, los cuales codifican para las proteínas denominadas receptores del factor de crecimiento transformante beta (TGFR- $\beta$ ), cuya función es la regulación de la transmisión de señales desde la superficie al interior celular $[13,14]$ y para los cuales se han descrito más de 30 y 80 mutaciones, que dan lugar a un receptor con poca o ninguna función [4]. El tipo 3 presenta una alteración en el gen SMAD3 (15q22.33) que codifica para una proteína implicada en la vía de señalización del factor de crecimiento transformante beta (TGF- $\beta$ ) [15], en el cual se han encontrado más de 10 mutaciones que generan una proteína SMAD3 no funcional [4]. En el síndrome de Loeys-Dietz tipo 4, el gen TGFB2 (1q41) es el que se encuentra alterado. Este gen codifica para el factor de crecimiento transformante beta-2 (TGF- $\beta 2$ ) [16]; al menos 14 mutaciones dan lugar a la codificación de una proteína TGF- $\beta 2$ con poca o ninguna función [4]. Finalmente, el tipo 5 del síndrome de Loeys-Dietz, descrito en 2013 por Rienhoff y colaboradores [12], es causado por una mutación en el gen TGFB3 (14q24.3). Esta variante se caracteriza por la presentación sindrómica de aneurismas aórticos a nivel torácico o abdominal, además de la presencia de paladar hendido, úvula bífida, enfermedad de la válvula mitral, crecimiento excesivo del esqueleto e inestabilidad de la columna cervical. Cabe resaltar que no todas las características clínicas ocurren en todos los pacientes [11,12].

El objetivo de esta publicación es presentar el primer caso de síndrome de Loeys-Dietz asociado a una mutación en el gen TGFBR2 en el suroccidente colombiano.

\section{Presentación de caso}

\section{Caso clínico}

Paciente masculino de 22 meses, producto del primer embarazo de una madre de 24 
años y un padre de 25 años, sin consanguinidad. Embarazo programado con ocho controles prenatales y dos amenazas de aborto a las 7 y 13 semanas de gestación, manejados con óvulos de progesterona. Percepción de movimientos fetales a las 13 semanas. El embarazo transcurrió en el casco urbano de Popayán, al suroccidente de Colombia, sin exposición a teratógenos. El parto fue inducido a las 38 semanas por diagnóstico prenatal de cardiopatía congénita realizado a las 22 semanas, sospecha de trisomía (pliegue nucal aumentado) y amniocentesis cariotipo $46 \mathrm{XY}$. Parto institucional en cuarto nivel, peso al nacer $2.870 \mathrm{~g}$, talla $51 \mathrm{~cm}$, perímetro cefálico $36 \mathrm{~cm}$.

El 8 de abril de 2015, en el momento del nacimiento, el paciente presentó necesidad de reanimación cerebro cardiopulmonar (RCCP). Mediante ecocardiograma transesofágico se evidencia estenosis crítica de la válvula pulmonar, displasia severa de la válvula tricúspide, displasia leve de la válvula mitral, aorta y arco elongados, además de ductus arterioso. El paciente es llevado a valvulotomía pulmonar de urgencia con necesidad de cuidado intensivo posoperatorio durante 27 días. Al examen físico se encuentra pie equino varo bilateral, camptodactilia y aracnodactilia. El paciente fue valorado por el servicio de Genética, el cual solicita panel NGS (del inglés, Next Generation Sequencing) para el síndrome de Marfan y otros relacionados. El 1 de junio de 2015 se indicó egreso con seguimiento ambulatorio y manejo multidisciplinario.

El 25 de junio de 2015 es valorado por oftalmología pediátrica y no se encuentra subluxación del cristalino ni otros signos oculares compatibles con el síndrome de Marfan, por lo que se indica seguimiento. El 28 de julio de 2015 se realiza control ecocardiográfico y se encuentra displasia valvular total, insuficiencia pulmonar leve sin estenosis residual, además de ductus arterioso restrictivo en vías de cierre espontáneo y fisiología de disfunción diastólica del ventrículo derecho. El 18 de diciembre de 2015 se realiza ecocardiograma en el cual se encuentra displasia de la válvula mitral, dilatación severa de la aorta a nivel de los senos de valsalva y dilatación homogénea de toda la aorta.

El 26 de abril de 2016 es valorado en el Hospital Universitario San José, de la ciudad de Popayán, Colombia, por Genética Clínica. En el examen físico se observó hábito longilíneo, marfanoide. Los signos vitales fueron: frecuencia cardiaca 80 latidos por minuto, tensión arterial $80 / 50 \mathrm{mmHg}$ (percentil para el paciente), frecuencia respiratoria 28 respiraciones por minuto, temperatura 37 ${ }^{\circ} \mathrm{C}$, saturación de oxígeno $96 \%$ aire ambiente. Los datos antropométricos fueron: peso $11 \mathrm{~kg}$, talla $88 \mathrm{~cm}$. Brazada $85 \mathrm{~cm}$, perímetro cefálico $50,5 \mathrm{~cm}$, perímetro torácico 49 $\mathrm{cm}$, perímetro abdominal $42 \mathrm{~cm}$, distancias intercánticas $85 \times 27 \mathrm{~mm}$, filtrum $11 \mathrm{~mm}$, pabellones auriculares $5 \times 2,5 \mathrm{~cm}$ bilateral, distancia intermamilar $11 \mathrm{~cm}$, segmento inferior $38 \mathrm{~cm}$, relación de segmentos 1,1, relación brazada-talla $-3 \mathrm{~cm}$.

Además, se observó cráneo dolicocéfalo, cabello fino hipopigmentado, frente prominente, cejas poco pobladas, pestañas largas y rectas, hendiduras palpebrales antimongoloides amplias y comisuras bucales dirigidas hacia abajo. Cara ovalada, larga, microretrognatia, paladar ojival, pabellones hiperlaxos con antihélix hipoplásico. Cuello largo y tórax con cicatriz quirúrgica antigua en parte posterior del hemitórax izquierdo. Soplo sistólico eyectivo en focos pulmonar y aórtico. Osteoarticular con pulgar que sobrepasa por el lado cubital con la mano em- 


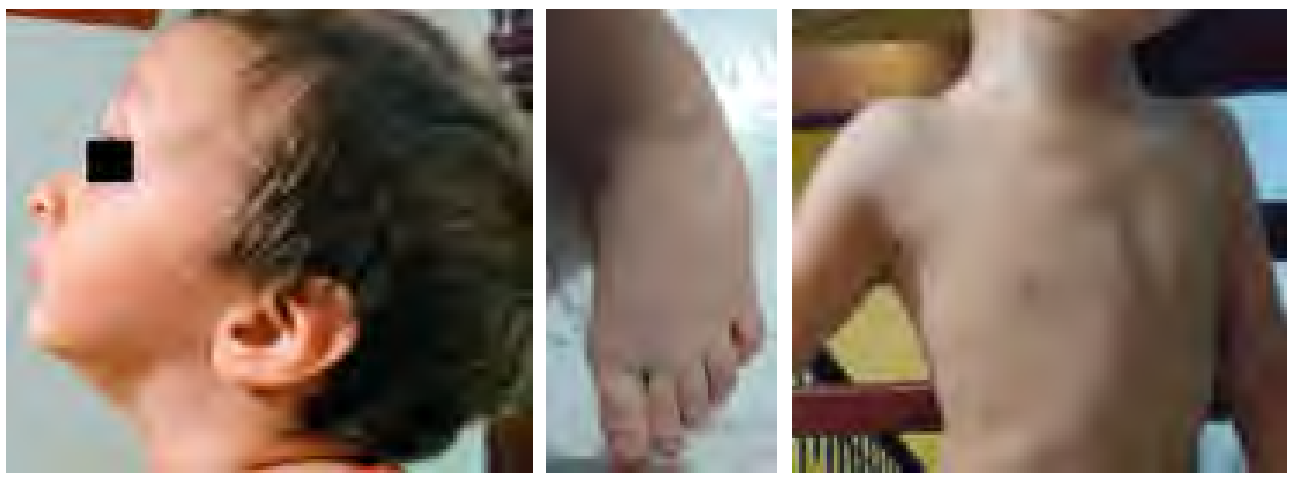

Figura 1. Fotografías del paciente. Véase descripción en el texto.

puñada, aranodactilia, hiperextensión pasiva articular, hiperflexión activa de dedos de más de 90 grados, dorsiflexión pasiva de la muñeca a 90 grados o más. Escápulas aladas, piel fina y delgada (véase figura 1).

Los resultados de paraclínicos solicitados muestran una mutación en el gen TGFBR2 (véase figura 2). Se solicita realizar estudio de cosegregación de esta mutación en la familia, además de control con cardiología pediátrica y test de Breighton (positivo para hiperlaxitud ligamentaria). El 13 de febrero de 2017 mostró un ecocardiograma de control con dilatación importante de la raíz aórtica de $36 \mathrm{~mm}$ de diámetro y arco aórtico elongado de 9 a $10 \mathrm{~mm}$ de diámetro. No se observa coartación en el ecocardiograma TT (véase figura 3 ). Se decide llevar el caso a junta cardioquirúrgica para definir el plan de manejo.

\section{Estudio molecular}

El estudio molecular se realizó mediante método de secuenciación masiva en paralelo utilizando una biblioteca que incluye 35 genes (ACTA2, ADAMTSL4, B3GAT3, CBS, COL1A1, COL1A2, COL3A1, COL5A1, COL5A2, EFEMP2, ELN, FBN1, FBN2, FLNA, GAA, GATA5, HRAS, KCNJ8, MED12,
MYH11, MYLK, NKX2-5, NOTCH1, PLOD1, PRKG1, PTPN11, SKI, SLC2A10, SMAD3, SMAD4, TGFB2, TGFB3, TGFBR1, TGFBR2 y ZDHHC9) relacionados con síndromes aórticos vasculares. Se identificó una mutación en el gen TGFBR2, que explica el fenotipo descrito y está asociada al fenotipo marfanoide y al síndrome de Loeys-Dietz (véase figura 2).

\section{Estudio bioinformático}

En el paciente en estudio se encontró la variante p.Leu427Pro en el gen TGFBR2, mediante un sistema bioinformático desarrollado por Health in Code (La Coruña, España), el cual permite identificar grandes inserciones/delecciones que afectan uno o más exones de alguno de los genes incluidos en el panel CNVs (del inglés, Copy Number Variations) y que presenta una sensibilidad y especificidad analíticas mayores al 99\%, según datos del laboratorio.

\section{Aspectos éticos}

Para la publicación de este caso clínico, en conjunto con sus fotografías, se obtuvo consentimiento informado de la madre, quien autorizó su divulgación con fines académicos. 


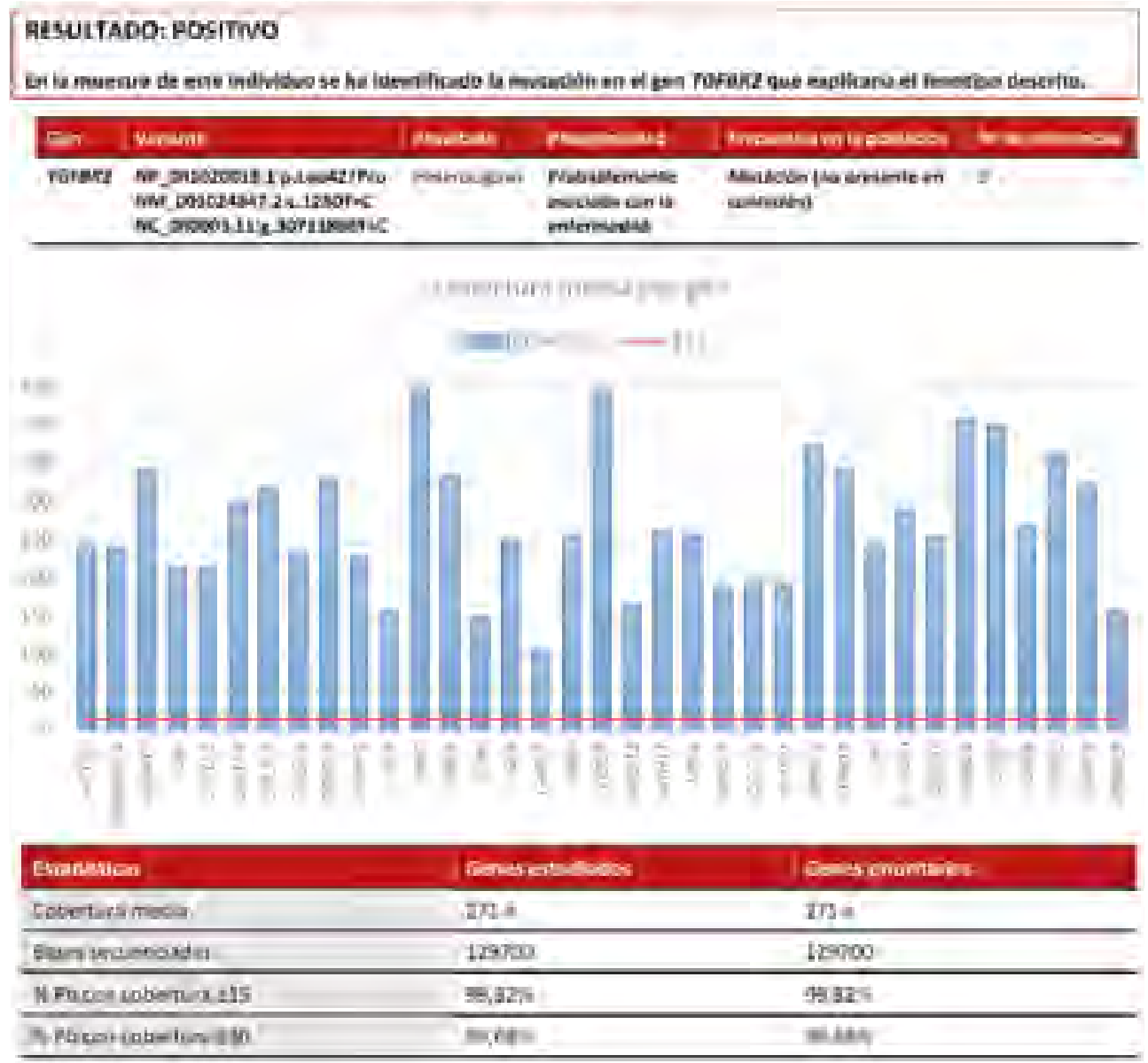

Figura 2. Resultado del estudio molecular del caso emitido por Health In Code, donde se muestra la mutación en el gen TGFBR2.

\section{Discusión}

Este artículo describe el primer caso clínico con síndrome de Loeys-Dietz asociado a una mutación en el gen TGFBR2 en el suroccidente colombiano, atendido en el Hospital Universitario San José de Popayán, Cauca (Colombia) en el segundo semestre de 2015. El síndrome de LoeysDietz es una enfermedad autosómica dominante que afecta principalmente el tejido conectivo [3,5], como se mencionó previamente. En un $75 \%$ de los casos se puede encontrar como una mutación de novo, lo cual resulta de vital importancia a la hora de brindar un correcto asesoramiento genético [4]. Esta enfermedad es causada por mutaciones en los genes TGFBR1, TGFBR2, TGFB2, TGFB3 y SMAD3 [4,9-12]. Aunque su prevalencia exacta es desconocida, en el $95 \%$ de los casos se encuentran asociadas a las mutaciones en los genes TGFBR2 y TGFBR1 $[5,8,11,13]$, como en el actual caso presentado. 


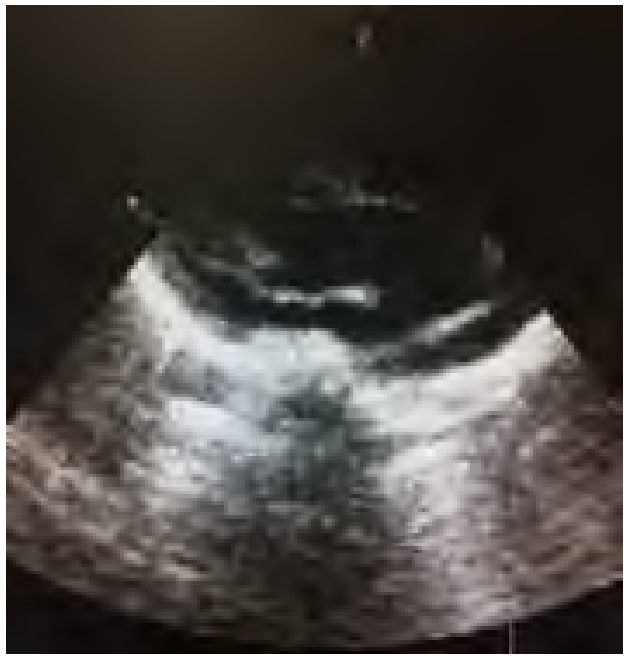

Figura 3. Ecocardiograma TT del caso. No se observa coartación.

El gen TGFB2 codifica para el factor de crecimiento transformante beta-2 (TGF- $\beta 2$ ), el cual se encuentra presente en todo el organismo. Para cumplir con sus funciones el TGF- $\beta 2$ se une a sus receptores en la superficie celular, los cuales son codificados por los genes TGFBR1 y TGFBR2 [4]. Específicamente, el TGFBR2 pertenece a la familia de quinasas de serina / treonina transmembrana y consiste en un ectodominio $\mathrm{N}$-terminal de unión al ligando, una región transmembrana y un dominio de serina / treonina quinasa en la región C-terminal. El ectodominio está formado por nueve hebras beta y una única hélice alfa estabilizada por una red de seis enlaces disulfuro intra-hilos [17].

La unión entre el TGF- $\beta$ y los receptores TGFBR1 y TGFBR2 lleva a la transmisión de señales en el interior de la célula y el control de diversas actividades celulares. El TGF- $\beta 2$ es crucial en la formación de los vasos sanguíneos, la regulación del tejido muscular, el desarrollo de la grasa corporal, la cicatrización de heridas y la función del sistema inmunológico [4].
El gen TGFBR2 fue aislado por primera vez en 1992 por Lin y colaboradores [18]; desde entonces, múltiples autores han trabajado en la búsqueda de diversas mutaciones asociadas a este gen (TGFBR2- síndrome de Loeys-Dietz tipo 2). Boileau y colaboradores (1993) [19] describieron una familia francesa con un trastorno del tejido conectivo, y algunas de las características esqueléticas y cardiovasculares que se observan en el síndrome de Marfan. Mizuguchi y colaboradores (2004) [20] identificaron la mutación 1524G-A en el gen TGFBR2, que explicaría años más tarde el fenotipo presentado en la familia francesa descrita por Boileau y colaboradores [19]; además, mencionan otras tres mutaciones (923T-C, 1346C-T y 1609CT) asociadas con el fenotipo característico, para ese entonces, del síndrome de Marfan tipo 2 [20].

Loeys y colaboradores (2005) [2] estudiaron 10 familias con un trastorno caracterizado por defectos cardiovasculares, craneofaciales, neurocognitivos y del desarrollo óseo, a los que le realizaron secuenciación de todos los exones del gen TGFBR2, donde encontraron diversas mutaciones heterocigotas (transversión 1006T-A en el exón 4, transversión 1063G-C en exón 4, transversión 1069G-T en exón 4, transición 1583G-A en exón 7, transición 1582C-T en exón 7 y transición 952A-G posición -2 en el intrón 1) en seis de las 10 familias. Las otras cuatro familias presentaron mutaciones en el gen TGFBR1. Por su parte, Pannu y colaboradores (2005) [21] llevaron a cabo un estudio en 80 familias no relacionadas con casos de aneurisma y disección de la aorta torácica, en las que lograron la secuenciación del cromosoma 3p25-p24 y detectaron, en cuatro de las familias, las transiciones $1378 \mathrm{C}-\mathrm{T}$ (dos familias) y 1379G-A (dos familias) en el exón 5 de este gen. 
En 2006, Disabella y colaboradores [22], y Law y colaboradores [23] describieron la misma mutación encontrada previamente por Pannu y colaboradores (R460H) [21]. Loeys y colaboradores (2006) [5] describieron otras dos alteraciones genéticas asociadas con el síndrome de Loeys-Dietz tipo 2, las mutaciones sin sentido pro 427 a Leu (P427L) y arg 495 a codón de parada (R495X). Kirmani y colaboradores (2010) [24] encontraron dos mutaciones heterocigotas en el gen TGFBR2, una transición C923T >C en el exón 4 y una C.1561T >C en el exón 7, en dos pacientes masculinos de 17 y 26 años, respectivamente [24].

Para el 8 de abril de 2016, en la versión en línea del proyecto Herencia Mendeliana en el Hombre (OMIM, del inglés, Online Mendelian Inheritance in Man) de la Universidad Johns Hopkins, se habían descrito un total de 19 variantes alélicas para el gen TGFBR2 [13]. Zimmermann y colaboradores (2017) [10] describen una variante patogénica en el exón 4, denotado por c.1061T>C p.(L354P) en una paciente de 10 años de edad.

De acuerdo con el reporte del estudio genético del paciente del presente caso, emitido por Health In Code, la variante p.Leu427Pro en el gen TGFBR2 encontrada no ha sido descrita previamente en ninguna publicación científica y tampoco aparece recogida en las bases de datos públicas que incluyen la información genética de individuos de diferentes etnias, que fueron utilizados como población control en la prueba genética solicitada [dbSNP del NCBI, NHLBI GO Exome Sequencing Project (ESP), Exome Aggregation Consortium (ExAC)].

Además, como resultado del estudio bioinformático, indican que el residuo de leucina 427 pertenece al dominio serina / treonina quinasa en la región C-terminal del receptor del TGF- $\beta 2$ (TGFBR2), y que en la proximidad de la sustitución de la leucina 427 por una prolina, se ha descrito la fosforilación de las serinas 434 y 441 y que, específicamente, la fosforilación de la serina 434 es esencial para la señalización quinasa del receptor del TGF- $\beta 2$. Finalmente, señalan que la variante $p$.Leu427Pro del paciente de nuestro caso produce el cambio de un aminoácido con cadena lateral hidrofóbica alifática, la leucina (Leu; CTG), por otro aminoácido con cadena lateral hidrofóbica cíclica, la prolina (Pro; CCG), lo que lleva a moderadas modificaciones en algunas propiedades fisicoquímicas (p. ej. polaridad, carga y volumen) entre la leucina y la prolina (distancia Grantham: 98 [0-215]).

\section{Conclusiones}

Este es el primer caso compatible con el síndrome de Loeys-Dietz en el suroccidente colombiano, en el que se identifica una mutación heterocigota c.1280T>G en el exón 5 del gen TGFBR2, lo que resulta en la sustitución de aminoácidos p.leu427pro (L427P), probablemente patogénica y quizás la única reportada en el mundo hasta el momento.

\section{Agradecimientos}

A la Universidad del Cauca y su Departamento de Pediatría (Popayán, Colombia).

\section{Bibliografía}

1. Furlong J, Kurczynski TW, Hennessy JR. New Marfanoid syndrome with craniosynostosis. Am J Med Genet 1987; 26: 599-604.

2. Loeys BL, Chen J, Neptune ER, Judge DP, Podowski M, Holm T, et al. A syndrome of altered cardiovascular, craniofacial, neurocognitive and skeletal development caused by mutations in TGFBR1 or TGFBR2. Nat Genet 2005; 37: 275281. 
3. Jamsheer A, Henggeler C, Wierzba J, Loeys B, De Paepe A, Stheneur C, et al. A new sporadic case of early-onset Loeys-Dietz syndrome due to the recurrent mutation p.R528C in the TGFBR2 gene substantiates interindividual clinical variability. J Appl Genet 2009; 50: 405-410.

4. Instituto Valenciano de Microbiología. Pruebas ge néticas - Loeys Dietz, Síndrome de... (Loeys Dietz, Syndrome) - Genes TGFBR1, TGFBR2, SMAD3, TGFB2 TGFB3. 2009. Disponible: http://www.ivami.com/es/ pruebas-geneticas-mutaciones-de-genes-humanos-enfermedades-neoplasias-y-farmacogenetica/5155-pruebasgeneticas-loeys-dietz-sindrome-de-loeys-dietz-syndromegenes-i-tgfbr1-tgfbr2-smad3-tgfb2-i-y-i-tgfb3-i. Consultado: feb 2017.

5. Loeys BL, Schwarze U, Holm T, Callewaert BL, Thomas GH Pannu H, et al. Aneurysm syndromes caused by mutations in the TGF-beta receptor. N Engl J Med 2006; 355: 788-798.

6. Fortuny E, Cañadas V, Vilacosta I. Aneurisma aórtico en síndromes hereditarios. Diagnóstico diferencial con el síndrome de Marfan. Cardiocore 2011; 46: 105-108.

7. Loeys BL, Dietz HC. Loeys-Dietz Syndrome. En: Pagon $\mathrm{RA}$, Adam MP, Ardinger $\mathrm{HH}$, Wallace SE, Amemiya A, Bean LJ, et al., eds. GeneReviews ${ }^{\circledR}$. Seattle, Estados Unidos: University of Washington; 1993-2017.

8. de León Ojeda NE, García García A. Síndrome Loeys Dietz. Presentación de una familia. Rev Cubana Genet Comunit 2008; 2: 61-64.

9. MacCarrick G, Black JH, 3rd, Bowdin S, El-Hamamsy I, Frischmeyer-Guerrerio PA, Guerrerio AL, et al. Loeys-Dietz syndrome: a primer for diagnosis and management. Genet Med 2014; 16: 576-587.

10. Zimmermann MT, Urrutia RA, Blackburn PR, Cousin MA, Boczek NJ, Klee EW, et al. Novel Pathogenic Variant in TGFBR2 Confirmed by Molecular Modeling Is a Rare Cause of Loeys-Dietz Syndrome. Case Rep Genet 2017; 2017 7263780 .

11. Online Mendelian Inheritance in Man ${ }^{\circledR}$. Loeys-Dietz Syndrome 5; LDS5. 2017. Johns Hopkins University. Dis ponible: https://www.omim.org/entry/615582?search=TG FB3\&highlight=tgfb3. Consultado: feb 2017.

12. Rienhoff HY, Jr., Yeo CY, Morissette R, Khrebtukova I, Melnick J, Luo S, et al. A mutation in TGFB3 associated with a syndrome of low muscle mass, growth retardation, distal arthrogryposis and clinical features overlapping with Marfan and Loeys-Dietz syndrome. Am J Med Genet A 2013; 161A: 2040-2046.

13. Online Mendelian Inheritance in Man $^{\circledR}$. Transforming
Growth Factor-Beta Receptor, Type II; TGFBR2. 2017. Johns Hopkins University. Disponible: https://www.omim.org/ entry/190182\#geneStructure. Consultado: feb 2017.

14. Online Mendelian Inheritance in Man ${ }^{\circledR}$. Transforming Growth Factor-Beta Receptor, Type I; TGFBR1. 2017. Johns Hopkins University. Disponible: https://www.omim.org/entry/190181. Consultado: feb 2017

15. Online Mendelian Inheritance in Man ${ }^{\circledR}$. Loeys-Dietz Syndrome 3; LDS3. 2017. Johns Hopkins University. Disponible: https://www.omim.org/entry/613795. Consultado: feb 2017

16. Online Mendelian Inheritance in Man ${ }^{\circledR}$. Transforming Growth Factor, Beta-2; TGFB2. 2017. Johns Hopkins University. Disponible: https://www.omim.org/entry/190220. Consultado: feb 2017

17. Hart PJ, Deep S, Taylor AB, Shu Z, Hinck CS, Hinck AP. Crystal structure of the human TbetaR2 ectodomain--TGF-beta3 complex. Nat Struct Biol 2002; 9: 203208.

18. Lin HY, Wang XF, Ng-Eaton E, Weinberg RA, Lodish HF. Expression cloning of the TGF-beta type II receptor, a functional transmembrane serine/threonine kinase. Cell 1992; 68: 775-785.

19. Boileau C, Jondeau G, Babron MC, Coulon M, Alexandre JA, Sakai L, et al. Autosomal dominant Marfan-like connective-tissue disorder with aortic dilation and skeletal anomalies not linked to the fibrillin genes. Am J Hum Genet 1993; 53: 46-54.

20. Mizuguchi T, Collod-Beroud G, Akiyama T, Abifadel M, Harada N, Morisaki T, et al. Heterozygous TGFBR2 mutations in Marfan syndrome. Nat Genet 2004; 36: 855-860.

21. Pannu H, Fadulu VT, Chang J, Lafont A, Hasham SN, Sparks E, et al. Mutations in transforming growth factor-beta receptor type II cause familial thoracic aortic aneurysms and dissections. Circulation 2005; 112: 513-520.

22. Disabella E, Grasso M, Marziliano N, Ansaldi S, Lucchell C, Porcu E, et al. Two novel and one known mutation of the TGFBR2 gene in Marfan syndrome not associated with FBN1 gene defects. Eur J Hum Genet 2006; 14: 34-38.

23. Law C, Bunyan D, Castle B, Day L, Simpson I, Westwood G, et al. Clinical features in a family with an $\mathrm{R} 460 \mathrm{H}$ mutation in transforming growth factor beta receptor 2 gene. J Med Genet 2006; 43: 908-916.

24. Kirmani S, Tebben PJ, Lteif AN, Gordon D, Clarke BL, Hefferan TE, et al. Germline TGF-beta receptor mutations and skeletal fragility: a report on two patients with Loeys-Dietz syndrome. Am J Med Genet A 2010; 152A: 1016-1019.

\begin{abstract}
Loeys-Dietz syndrome is a rare, autosomal dominant genetic disease, with marfanoid habit, which belongs to a subset of diseases of the connective tissue with mainly skeletal, ocular, and cardiovascular involvement. Aneurysms development is characteristic in this pathology. Loeys-Dietz syndrome is caused by mutations in TGFBR1, TGFBR2, TGFB2, TGFB3 and SMAD3 genes. In this manuscript is presented the clinical case of a 22-month-old male patient with significant dilatation of the aortic root and elongated aortic arch is described. The molecular test confirms the diagnosis of Loeys-Dietz syndrome associated with a mutation in the TGFBR2 gene. This corresponds to the first case reported in the southwestern Colombian.
\end{abstract}

Keywords: Loeys-Dietz syndrome, transforming growth factor-beta type II receptor, mutation, case management. 